\title{
PROPUESTA DE LÍMITES DE EXPOSICIÓN OCUPA- CIONAL PARA LA PROTECCIÓN CONTRA CAMPOS ELECTROMAGNÉTICOS DE LOS SISTEMAS DE RESONANCIA MAGNÉTICA
}

Victor Cruz Ornetta

\section{Resumen}

El objetivo del presente estudio fue formular una propuesta de límites para la exposición ocupacional a los campos electromagnéticos producidos por los equipos de Imaginología por Resonancia Magnética (IRM). Para ello se realizó una revisión de la literatura científica respecto de los niveles de exposición provocados, los posibles efectos, las recomendaciones internacionales relevantes y finalmente se propuso los límites de exposición ocupacionales.

Como resultado se han definido los principales posibles efectos sobre la salud de las personas. Se realizó una comparación entre diferentes recomendaciones internacionales sobre el tema y se formuló la recomendación de límites de exposición ocupacionales peruanos.

Palabras clave: Resonancia magnética, IRM, exposición ocupacional, ICNIRP, OMS, gradiente de campo magnético

\section{Abstract}

The objective of this study was to make a proposal of limits for occupational exposure to electromagnetic fields from Magnetic Resonance Imaging (MRI) equipment. In order to perform this task, it was carried out a revision of scientific literature on exposure levels, posible health effects and international guidelines, then it was prepared the proposal of occupational limits. As a result of this work it was defined the main posible health effects, it was made a comparisson of different international guidelines and it was finally produced the proposal of the Peruvian occuptional limits on MRI.

Keywords: Magnetic resonance, MRI, occupational exposure, ICNIRP, WHO, magnetic field gradient 


\section{INTRODUCCIÓN}

La Imaginología por Resonancia Magnética es una técnica de diagnóstico utilizada para obtener imagenes de alta calidad, basada en la absorción o liberación de energía electromagnética por los núcleos atómicos del cuerpo humano inmersos en un campo estático después de la excitación por un pulso de radiofrecuencia. Un potente magneto que puede ser permanente, resistivo o superconductor, genera un campo magnético alrededor de 10000 a 100000 veces más fuerte que el campo magnético natural de la Tierra (aproximadamente $50 \mu \mathrm{T}$ ). Actualmente, varios tipos de sistemas de resonancia magnética clínica utilizan magnetos superconductores que generan campos magnéticos de 0.5 a 3.0 $\mathrm{T}$ y se han desarrollado sistemas con propósitos de investigación con campos aún más altos ( $\geq 7 \mathrm{~T})$. Asimismo, el sistema utiliza gradientes de campos magnéticos para realizar la exploración de la zona objetivo del cuerpo y campos de radiofrecuencia para llegar a la resonancia $(1,2)$.

A diferencia de la radiografia convencional y la imaginología por tomografia computarizada, las cuales hacen uso de radiación ionizantes potencialmente dañina (rayos X), la IRM tiene muchas ventajas, incluyendo su naturaleza no ionizante y su habilidad sin paralelo para discriminar entre diferentes tejidos blandos sin necesidad de un medio de contraste. De esta manera, la IRM se ha constituido en una modalidad establecida de diagnóstico por imagen. La utilidad clínica de la Resonancia Magnética Convencional e Intervencional cada vez es mayor; sin embargo, estas técnicas implican la exposición del paciente y del personal médico y paramédico a campos magnéticos estáticos y variables en el tiempo, que en condiciones particulares de exposición podrían implicar peligro para la salud o un incremento de riesgo. Aunque los análisis experimentales y teóricos apoyan la inocuidad de las interacciones entre los campos de los sistemas de IRM y el cuerpo humano, sin embargo, la literatura no demuestra la absoluta seguridad de la exposición prolongada a los campos magnéticos.

La Unión Europea (UE) emitió en el 2004 la Directiva 2004/40/EC sobre agentes fisicos (1), que incluyen los ruidos, vibraciones y campos electromagnéticos, entre otros. En el caso de los campos electromagnéticos, la directiva adopta los limites recomendados por la Comisión de Protección contra las Radiaciones No Ionizantes como "Valores Límites de Exposición (ELV)", basados directamente en los efectos sobre la salud conocidos y en consideraciones biológicas cuyo cumplimiento garantizará que los trabajadores expuestos a campos electromagnéticos estén protegidos contra todo efecto nocivo conocido para la salud, que no pueden ser excedidos bajo ninguna circunstancia; y suplementariamente, los "Valores de Acción (AV)", que son los valores directamente medibles cuyo respeto asegura el cumplimiento de los límites. La transposición 
de la directiva a las legislaciones de los países miembros de la UE estaba prevista para el 30 de abril de 2008 a más tardar. Sin embargo, la Directiva 2008/46/EC (2), del 23 de abril de 2008, postergó la transposición para el 30 de abril de 2012 a más tardar; $y$, finalmente, mediante la Directiva 2012/11/UE (3), de 19 de abril de 2012, se amplía el plazo hasta el 31 de octubre de 2013

En los años 2010-2011, se realizó un estudio desarrollado por la Dirección General de Salud Ambiental del Ministerio de Salud (DIGESA-n MIN$\mathrm{SA}$ ), conjuntamente con la Universidad Nacional Mayor de San Marcos (FIEE- UNMSM) y la Universidad de Ingeniería (INICTEL-UNI y FIA-UNI), sobre la exposición ocupacional a campos electromagnéticos producida por los equipos de terapia de pulsos, diatermia terapéutica y electrocirugía. En dicho estudio, no se tomó en cuenta a los equipos de resonancia magnética por representar una problemática más compleja, y se decidió abordarlo más adelante

Estos antecedentes demuestran la preocupación internacional y peruana por establecer límites adecuados para la exposición ocupacional a los campos electromagnéticos de los equipos IRM que, sin embargo, de acuerdo a la experiencia europea, no son fáciles de establecerlos.

Los estimados iniciales muestran que para los trabajadores que realizan movimientos cerca del magneto, se superan los ELV por un factor de 5 a 10 veces para campos magnéticos con frecuencias en el orden de $1 \mathrm{~Hz}$ $\mathrm{y}$, cuando los gradientes conmutados están operando, la exposición probablemente excede los ELV para campos magnéticos de $500-1000 \mathrm{~Hz}$ por un factor del orden de $20(4,8)$. En general, los ELV para radiofrecuencia de la Directiva 2004/40/EC pueden ser cumplimentados para cualquiera de los procedimientos actuales, excepto cuando dos personas están simultáneamente dentro de la cavidad cilíndrica del sistema. Cualquier superposición de cuerpos debería ser evitada, ya que puede llevar a exposiciones mucho más altas que exceden considerablemente las recomendaciones. Los procedimientos actualmente aplicados para IRM intervencional resultan en valores de SAR, cercanos a los límites de SAR; sin embargo, la exposición podría ser minimizada con medidas apropiadas $(4,8)$.

Esta situación genera cierta preocupación con respecto a la exposición de los pacientes y del personal médico y paramédico a los campos electromagnéticos de la IRM. La exposición del paciente, generalmente, no está limitada por las recomendaciones internacionales, debido a que se considera que estará bajo supervisión, trasladando la responsabilidad al físico médico o al tecnólogo médico. En el caso del personal médico y paramédico, se deberán observar las recomendaciones internacionales que limitan la exposición de los trabajadores.

Es conveniente señalar que Directiva Europea establece los requerimientos mínimos, es decir, da a los esta- 
dos miembros la opción de mantener o adoptar provisiones más favorables para la protección de los trabajadores, en particular la fijación de valores más bajos para los valores de acción o los valores de los límites de exposición para campos electromagnéticos. La implementación de esta Directiva no justifica ningún retroceso en relación a la situación que prevalece en el Estado Miembro. El nivel de exposición a los campos electromagnéticos puede ser más efectivamente reducido, incorporando medidas preventivas en el diseño de las estaciones de trabajo y seleccionando el equipamiento, procedimientos y métodos para priorizar la reducción de riesgos en la fuente. Por lo tanto, las provisiones deberían incluirlas, pues contribuyen a la protección de los trabajadores. Los empleadores deberian hacer ajustes a la luz del progreso técnico y el conocimiento científico respecto de los riesgos relacionados con la exposición a campos magnéticos, con el objetivo de mejorar la protección de la seguridad y salud de los trabajadores involucrados.

En concordancia con lo expuesto, el presente estudio tiene como objetivo plantear una propuesta de limites máximos de exposición ocupacionales a ser aplicados en el Perú.

\section{MATERIALES Y MÉTODOS}

Para este estudio se ha realizado una revisión bibliográfica de los documentos más importantes publicados en los últimos años sobre los efectos en la salud, la evaluación de los cam- pos electromagnéticos y la regulación internacional referente a los sistemas de imaginología por resonancia magnética. En base a estos documentos, se plantea la propuesta de límites máximos de exposición peruanos.

\section{RESULTADOS Y DISCUSIONES}

\section{Efectos sobre la salud de las personas Campo estático}

Los mecanismos plausibles para posibles efectos agudos sobre la salud por la exposición a los campos magnéticos estáticos asociados a la IRM, principalmente involucran las diferencias en la susceptibilidad magnética entre tejidos y la inducción de campos eléctricos y corrientes internos, los cuales pueden tener lugar cuando el cuerpo entero se mueve en un campo estático o cuando tejidos eléctricamente conductores se mueven en el cuerpo (p. ej. la sangre, el corazón). Si son lo suficientemente altos estos campos internos, podrian interferir con la función de tejidos eléctricamente excitables, tales como el corazón y los nervios, o inducir fuerzas que se oponen al flujo de la sangre desde el corazón a los tejidos del cuerpo (7). Al respecto, ningún efecto sobre el ritmo cardiaco, arritmias cardiacas o el flujo de sangre que puedan plantear un riesgo de salud, han sido reportados cerca de magnetos de hasta $8 \mathrm{~T}(8,9)$.

El movimiento de voluntarios en la cavidad de un magneto de cuerpo entero de $7 \mathrm{~T}$, a una velocidad de 0.1 $\mathrm{m} \mathrm{s}^{-1}$, resultó en una sensación de rotación en algunos sujetos, encontrán- 
dose que la probable causa sería una corriente inducida en la salida neural del sistema vestibular. En estudios en animales y en voluntarios, se ha observado que los movimientos de la cabeza dentro de un campo homogéneo (gradiente cero) pueden causar vértigo de magnitud pequeña a severa, náuseas que pueden durar hasta 30 minutos y sabor metálico, probablemente debido a la diferencia de susceptibilidades magnéticas entre la mácula del oído interno y sus alrededores, efectos magnetohidrodinámicos sobre la endolinfa del aparato vestibular y la estimulación eléctrica de la tasa de disparo (9). Asimismo, se ha observado la producción de magnetofosfenos, posiblemente debido a la estimulación de las terminaciones nerviosas en la retina del ojo $(8,10)$.

Hay alguna evidencia de una reducción menor al $5 \%$ en la concentración, memoria, velocidad y rendimiento visualmotor después del movimiento cerca de magnetos de 1.5-7 $(8,10)$, lo que podría afectarla calidad de la intervención quirúrgica o cualquier otra tarea realizada cerca de la cavidad del magneto.

\section{Gradientes conmutados de campo}

Los gradientes conmutados de campo no son sinusoidales sino pulsantes. Su intensidad crece rápidamente durante el pulso y disminuye a cero entre los pulsos.

El único efecto biológico descrito por ICNIRP $(4,5)$, que ha sido reportado para frecuencias mayores a $60 \mathrm{~Hz}$, es la estimulación nerviosa periférica
(SNP), la cual ocurre cuando los nervios sensoriales son estimulados por corrientes eléctricas inducidas por campos magnéticos variables en el tiempo, tales como los gradientes de campo. Ello resulta en una sensación que va desde el hormigueo al dolor intolerable, dependiendo de la amplitud del campo y su forma severa indudablemente es un efecto adverso sobre la salud. Sin embargo, el umbral de densidad de corriente para efectos sobre el SNP, aun en su forma más leve, es alrededor de $1 \mathrm{Am}^{-2}, 100$ veces el límite de exposición de la Directiva. E1 Estándar de la Comisión Electrotécnica Internacional (IEC), que gobierna la fabricación de los sistemas IRM, está diseñado para evitar los efectos sobre el SNP en pacientes y trabajadores.

Por razones prácticas, cuando se realiza una exploración de RM, el operador normalmente opera el escáner desde una sala de control separada; sin embargo, hay circunstancias en las cuales un miembro del staff permanece en la sala de exploración y cerca al escáner mientras está operando, y que en el caso del campo magnético estático y de los gradientes de campos magnéticos podrian implicar violaciones a los límites de exposición $(1,2)$ :

- IRM intervencional, donde un radiólogo u otro médico puede ser expuesto dentro de la cavidad del magneto para realizar procedimientos invasivos durante la exploración.

- Algunos tipos de IRM funcional, tales como estudios de investigación en sujetos sordos o ciegos donde un 
miembro del staff toca la palma de la mano del paciente durante el escaneo.

- Exámenes de niños donde la presencia cercana de la enfermera o radiólogo puede evitar la necesidad de anestesia para obtener imágenes satisfactorias.

- Exámenes de pacientes que están anestesiados o requieren monitoreo, casos en los cuales es común que un anestesiólogo permanezca en el salón para evaluar visualmente al paciente durante la exploración.

- Aplicaciones donde el investigador necesita ajustar experimentalmente el equipo durante el escaneo.

- Procedimientos de limpieza actuales que requieren que el personal gatee dentro de la cavidad del escáner.

La intensidad de la estimulación nerviosa depende de la intensidad del campo inducido (es decir de la tasa de cambio del campo magnético externo, $\mathrm{dB} / \mathrm{dt}$ ) y de la duración del pulso (2). La estimulación de los nervios sensoriales, dependiendo de la intensidad del campo, puede causar una sensación que va de un leve hormigueo (15 $\mathrm{Ts}^{-1}$ para un pulso de duración mayor a $1 \mathrm{~ms})$ al dolor intenso $\left(25 \mathrm{Ts}^{-1}\right) \mathrm{y}$ al dolor intolerable $\left(25 \mathrm{Ts}^{-1}\right)$. El umbral más bajo corresponde a un campo eléctrico interno de aproximadamente $5 \mathrm{Vm}^{-1}(2)$. Los nervios periféricos son más sensibles a la estimulación del gradiente de campo que los nervios del sistema nervioso central, porque sus diámetro es mayor y porque el campo eléctrico inducido en una persona echada dentro de un magneto es mayor en la periferia (extremidades) que en el núcleo del cuerpo (cerebro, espina dorsal)) (10). El umbral de gradiente $\mathrm{dB} / \mathrm{dt}$ para estimulación eléctrica del corazón es aproximadamente diez veces mayor a la estimulación nerviosa periférica (10).

\section{Campos de radiofrecuencia}

El principal riesgo de los CEM de radiofrecuencia es el calentamiento del cuerpo o de partes del cuerpo a un nivel para los cuales los mecanismos compensatorios normales son inadecuados y pueden ocurrir daños a las células o tejidos. Basados en los estudios en animales y voluntarios, el umbral para el daño de tejidos se estima que se da cuando la temperatura alcanza $1^{\circ} \mathrm{C}$, correspondiente a una tasa de absorción específica $4 \mathrm{Wkg}^{-1}$. Los órganos más sensibles del cuerpo son los ojos y los testículos, debido a la poca irrigación con que cuentan (4).

Los sistemas IRM con las densidades de flujo de campo magnético más altas requieren radiofrecuencias más altas. A estas frecuencias, la energía es acoplada más eficientemente al cuerpo y puede causar un calentamiento no uniforme ("hot spots") (6). Un lazo conductor con riesgo de quemaduras son las zonas de la piel, donde dos extremidades o extremidades y tronco hacen contacto. El cuerpo humano tiene una gran capacidad de disipación del calor; sin embargo, esta capacidad puede ser reducida por una temperatura ambiental más alta, medicación o enfermedad. (4). 


\section{REGULACIÓN INTERNACIONAL}

La Unión Europea, tomando en cuenta esta preocupación, ha emitido la Directiva 2004/40/EC del Parlamento y del Consejo Europeo sobre los requerimientos mínimos de salud y seguridad de los trabajadores a los riesgos provenientes de agentes físicos (campos electromagnéticos). Considera que es necesario introducir medidas de protección ocupacional contra riesgos asociados con los campos electromagnéticos de $0 \mathrm{~Hz}$ a $300 \mathrm{GHz}$, debido a sus efectos sobre la salud y la seguridad de los trabajadores de corto plazo establecidos, incluyendo los efectos causados por la circulación de corrientes inducidas (en el sistema nervioso central) y por la absorción de energía, así como por corrientes de contacto. Sin embargo, en esta Directiva no se abordan los efectos de largo plazo, incluyendo posibles efectos carcinogénicos debido a la exposición a campos eléctricos, magnéticos y electromagnéticos variables en el tiempo, para los cuales no existe evidencia científica concluyente, estableciendo una relación causal. La mencionada Directiva acoge las Recomendaciones ICNIRP 1998, planteando las Restricciones Básicas como "Valores Límites de Exposición (ELV)", los cuales no pueden ser excedidos bajo ninguna circunstancia, y suplementariamente los Niveles de Referencia, como los "Valores de Acción (AV)", los cuales aseguran el cumplimiento de los limites. En las tablas 1 y 2 se muestran los ELV y los AV (3).
Tabla 1. Valores limites de exposición. Han de cumplirse todas las condiciones

\begin{tabular}{|c|c|c|c|c|c|}
\hline$f$ & $\begin{array}{c}\mathrm{J} \\
\left(\mathrm{mA} \mathrm{m}^{-2}\right) \\
(\mathrm{rms})\end{array}$ & $\begin{array}{l}\mathrm{SAR}_{1} \\
\left(\mathrm{Wkg}^{-1}\right)\end{array}$ & $\begin{array}{l}\mathrm{SAR}_{2} \\
\left(\mathrm{Wkg}^{-1}\right)\end{array}$ & $\begin{array}{l}\mathrm{SAR}_{3} \\
\left(\mathrm{Wkg}^{-1}\right)\end{array}$ & $\begin{array}{c}S \\
\left(\mathrm{Wm}^{-2}\right)\end{array}$ \\
\hline $\begin{array}{ll}\text { hasta } & 1 \\
\mathrm{~Hz}\end{array}$ & 40 & -- & -- & -- & -- \\
\hline $1-4 \mathrm{~Hz}$ & $40 / f$ & -- & -- & -- & -- \\
\hline $\begin{array}{l}\begin{array}{l}4 \mathrm{~Hz}-1 \\
\mathrm{kHz}\end{array}\end{array}$ & 10 & -- & -- & -- & -- \\
\hline $\begin{array}{l}1-100 \\
\mathrm{kHz}\end{array}$ & $f / 100$ & -- & -- & -- & -- \\
\hline $\begin{array}{ll}0.1- & 10 \\
\mathrm{MHz} & \end{array}$ & $f / 100$ & 0.4 & 10 & 20 & -- \\
\hline $\begin{array}{ll}0.01 & -10 \\
\mathrm{GHz} & \end{array}$ & -- & 0.4 & 10 & 20 & -- \\
\hline $\begin{array}{l}10-300 \\
\mathrm{GHz}\end{array}$ & -- & -- & -- & -- & 50 \\
\hline
\end{tabular}

1. $f$ es la frecuencia en $\mathrm{Hz}$

2.J densidad de corriente para cabeza y tronco

3. $\mathrm{SAR}_{1}$ promedio en todo el cuerpo

4. $\mathrm{SAR}_{2}$ local cabeza y tronco

5. $\mathrm{SAR}_{3}$ local extremidades

6.S densidad de potencia

Tabla 2. Valores que dan lugar a una acción (valores rms imperturbados)

\begin{tabular}{lllll}
\hline \multicolumn{1}{c}{$f$} & $\begin{array}{c}\mathrm{E} \\
\left(\mathrm{Vm}^{-1}\right)\end{array}$ & \multicolumn{1}{c}{$\begin{array}{c}\mathrm{H} \\
\left(\mathrm{Am}^{-1}\right)\end{array}$} & $\begin{array}{c}\mathrm{B} \\
(\mu \mathrm{T})\end{array}$ & $\begin{array}{c}\mathrm{S} \\
\left(\mathrm{Wm}^{-2}\right)\end{array}$ \\
\hline Hasta $1 \mathrm{~Hz}$ & - & $1.63 \times 10^{5}$ & $2 \times 10^{5}$ & - \\
$1-8 \mathrm{~Hz}$ & 20000 & $1.63 \times 10^{5} / f^{2}$ & $2 \times 10^{5} / f^{2}$ & - \\
$8-25 \mathrm{~Hz}$ & 20000 & $2 \times 10^{4} / f$ & $2.5 \times 10^{4} / f$ & - \\
$0.025-0.82$ & & & & - \\
$\mathrm{kHz}$ & $500 / f$ & $20 / f$ & $25 / f$ & - \\
$0.82-65 \mathrm{kHz}$ & 610 & 24.4 & 30.7 & - \\
$0.065-1 \mathrm{MHz}$ & 610 & $1.6 / f$ & $2 / f$ & - \\
$1-10 \mathrm{MHz}$ & $610 / f$ & $1.6 / f$ & $2 / f$ & - \\
$10-400 \mathrm{MHz}$ & 61 & 0.16 & 0.2 & 10 \\
$400-2000 \mathrm{MHz}$ & $3 f 0.5$ & $0.008 f 0.5$ & $0.01 f 0.5$ & $f / 40$ \\
$2-300 \mathrm{GHz}$ & 137 & 0.36 & 0.45 & 50 \\
\hline
\end{tabular}

1. $f$ está en la frecuencia que se indica en la columna

2.E Intensidad de Campo Eléctrico

3.H Intensidad de Campo Magnético

4.B Densidad de Flujo Magnético

5.S Densidad de Potencia 
En la Tabla 3 se muestran las principales recomendaciones internacionales pertinentes $(12,13,14,15,16,17)$.

\begin{tabular}{|c|c|c|}
\hline Instútución & Aplicable a & Límites \\
\hline ICNIRP 1998 & $\begin{array}{l}\text { CEM variables hasta } \\
300 \mathrm{GHz} \text {, ocupacionaly } \\
\text { púublica en general }\end{array}$ & $\begin{array}{l}\text { Valores usados por la } \\
\text { Directiva 2004/40:EC }\end{array}$ \\
\hline IEEE 2003 & $\mathrm{CEM} 0 \mathrm{~Hz}-3 \mathrm{kHz}$ & $\begin{array}{l}\text { Ocupacional, campo estático } \\
353 \mathrm{mT}, 20-759 \mathrm{~Hz} 2.71 \mathrm{mT}\end{array}$ \\
\hline ICNIRP 2004 & $\begin{array}{l}\text { CEM para pacientes } \\
\text { IRM }\end{array}$ & $\begin{array}{l}4 \mathrm{~T} \\
\mathrm{~dB} / \mathrm{dt}=20(1+0.36 \mathrm{~T} / \mathrm{T} \mathrm{s}-1 \\
\text { para prevenir efectos sobre } \\
\text { SNP } \\
4 \mathrm{~W} / \mathrm{kg}\end{array}$ \\
\hline IEEE 2005 & $\begin{array}{l}\text { CEM } 3 \mathrm{kHz}-300 \mathrm{GHz} \\
\text { ocupacional y público } \\
\text { en general }\end{array}$ & $\begin{array}{l}\text { Ocupacional } 30-100 \mathrm{MHz} \text {, } \\
\text { 16.3/f(MHz) A/m; } \\
\text { 100-300MHz, } 0.163 \mathrm{Am}\end{array}$ \\
\hline $\begin{array}{l}\text { IEC 60601- } \\
2-33-2007\end{array}$ & $\begin{array}{l}\text { CEM para pacientes } \\
\text { IRM }\end{array}$ & $\begin{array}{l}2 \mathrm{~T} \text { modo controlado, } \\
4 \mathrm{~T} \text { limite } \\
\mathrm{dB} / \mathrm{dt} \text { umbral para SNP } \\
0.4 \mathrm{~W} / \mathrm{kg}\end{array}$ \\
\hline ICNIRP 2009 & $\begin{array}{l}\text { CM estáticos, } \\
\text { ocupacional y público } \\
\text { en general }\end{array}$ & $\begin{array}{l}\text { Ocupacional } 2 \mathrm{~T} \text { cabeza y } \\
\text { tronco, } 8 \mathrm{~T} \text { extremidades }\end{array}$ \\
\hline ICNIRP 2009 & $\begin{array}{l}\text { CEM para pacientes } \\
\text { IRM }\end{array}$ & $\begin{array}{l}\text { 4T para operación normal } \\
8 \mathrm{~T} \text { para operación en modo } \\
\text { controlado } \\
>8 \mathrm{~T} \text { en modo experimental }\end{array}$ \\
\hline ICNIRP 2010 & $\begin{array}{l}\text { CEM variables } 1 \mathrm{~Hz} \\
\text { hasta } 100 \mathrm{WHz}, \\
\text { ocupacional y púublica } \\
\text { en general }\end{array}$ & $\begin{array}{l}\text { Ocupacional, los límites se } \\
\text { dan en términos de los } \\
\text { campos eléctricos inducidos } \\
\text { internos }\end{array}$ \\
\hline
\end{tabular}

PROPUESTA DE LÍMITES MÁXIMOS DE EXPOSICIÓN PERUANOS

Los límites propuestos están basados en las Recomendaciones ICNIRP 1998, ICNIRP 2009 e ICNIRP 2010.

En la Tabla 4, se muestran los límites propuestos, mientras en las 5 y 6 se muestran los limites ICNIRP 2010 y 1998 relevantes a la norma propuesta.
Tabla 4. Limites de exposición ocupacional para IRM recomendados para el Perú

\begin{tabular}{lll}
\hline Tipo de campo & Frecuencia & $\begin{array}{l}\text { Límite de exposición } \\
\text { ocup. recomendado }\end{array}$ \\
\hline $\begin{array}{l}\text { Campo } \\
\text { magnético }\end{array}$ & $0 \mathrm{~Hz}$ & $\begin{array}{l}\text { ICNIRP 2009 } \\
\text { 4T operación nomal } \\
\text { estático (siempre }\end{array}$ \\
$\begin{array}{l}\text { presente en casión controlada } \\
\text { todos los casos) }\end{array}$ & $\begin{array}{l}<1 \mathrm{~Hz} \text { típico } \\
\text { (movimiento } \\
\text { de un sujeto) }\end{array}$ & ICNIRP 2010 \\
$\begin{array}{l}\text { Gradientes } \\
\begin{array}{l}\text { conmutados de de } \\
\text { campo magnético } \\
\text { (solo durante la }\end{array}\end{array}$ & $\begin{array}{l}\text { (típico) }-1 \mathrm{kHz} \\
\text { exploración) }\end{array}$ & ICNIRP 2010 \\
\end{tabular}

Tabla 5. Niveles de referencia para exposición ocupacional

a campos eléctricos y magnéticos variables en el tiempo

$1 \mathrm{~Hz}-10 \mathrm{MHz}$ (valores rms no peturbados) -ICNIRP 2010

\begin{tabular}{llll}
\hline \multicolumn{1}{c}{$f$} & \multicolumn{1}{c}{$\begin{array}{c}\mathrm{E} \\
\left(\mathrm{kVm}^{-1}\right)\end{array}$} & \multicolumn{1}{c}{$\begin{array}{c}\mathrm{H} \\
\left(\mathrm{Am}^{-1}\right)\end{array}$} & \multicolumn{1}{c}{$\begin{array}{c}\mathrm{B} \\
(\mathrm{T})\end{array}$} \\
\hline $1-8 \mathrm{~Hz}$ & 20 & $1.63 \times 10^{5 / f^{2}}$ & $0.2 / f^{2}$ \\
$8-25 \mathrm{~Hz}$ & 20 & $2 \times 10^{4 / f}$ & $2.5 \times 10^{-2} / f$ \\
$25-300 \mathrm{~Hz}$ & $500 / f$ & 800 & $1 \times 10^{-3}$ \\
$0.3-3 \mathrm{kHz}$ & $500 / f$ & $2.4 \times 10^{5 / f}$ & $0.3 / f$ \\
$3 \mathrm{kHz}-10 \mathrm{MHz}$ & 0.17 & 80 & $1 \times 10^{-4}$ \\
\hline
\end{tabular}

Tabla 6. Niveles de referencia para exposición ocupacional

a campos eléctricos y magnéticos variables en el tiempo

$10 \mathrm{MHz}-300 \mathrm{GHz}$ (valores rms no peturbados) -ICNIRP 1998

\begin{tabular}{lllll}
\hline \multicolumn{1}{c}{$f$} & $\begin{array}{c}\mathrm{E} \\
\left(\mathrm{Vm}^{-1}\right)\end{array}$ & \multicolumn{1}{c}{$\begin{array}{c}\mathrm{H} \\
\left(\mathrm{Am}^{-1}\right)\end{array}$} & $\begin{array}{c}\mathrm{B} \\
(\mu \mathrm{T})\end{array}$ & $\begin{array}{c}\mathrm{S} \\
\left(\mathrm{Wm}^{-2}\right)\end{array}$ \\
\hline $10-400 \mathrm{MHz}$ & 61 & 0.16 & 0.2 & 10 \\
$400-2000$ & $3 f^{0.5}$ & $0.008 f^{0.5}$ & $0.01 f^{0.5}$ & $f / 40$ \\
$\mathrm{MHz}$ & 137 & 0.36 & 0.45 & 50 \\
$2-300 \mathrm{GHz}$ & 137 & \\
\hline
\end{tabular}

\section{CONCLUSIONES}

Los limites de exposición ocupacional recomendados están basados en los niveles de referencia ICNIRP, que cubren básicamente todas las situaciones normales de un escaneo IRM y están actualizados al 2010. 


\section{REFERENCIAS BIBLIOGRAFÍCAS}

European Commission (2004). Directive 2004/40/EC of the European Parliament and of the Council of 29 April 2004 on the minimum health and safety requirements regarding the exposure of workers to the risks arising from physical agents (electromagnetic fields). Official Journal of the European Union L 159 of 30 April 2004, and corrigenda L 184 of 24 May 2004.

Directive 2008/46/EC of the European Parliament and of the Council (2008) amending Directive 2004/40/EC on minimum health and safety requirements regarding the exposure of workers to the risks arising from physical agents (electromagnetic fields) Official Journal of the European Union L 114.

Directiva 2012/11/UE del Parlamento Europeo y del Consejo (2012). Por la que se modifica la Directiva 2004/40/CE, sobre las disposiciones mínimas de seguridad y de salud relativas a la exposición de los trabajadores a los riesgos derivados de los agentes físicos (campos electromagnéticos) (decimoctava Directiva específica con arreglo al artículo 16 apartado 1, de la Directiva 89/391/ CEE: http://eur-lex.europa.eu/LexUriServ/LexUriServ.do?uri=OJ:L:2012:110: 0001:0002:ES:PDF

Keevil, S. (2008). MRI and Physical Agents (EMF) Directive [Internet]. Londres: Institute of Physics; Nov 2008 [citado 3 de junio de 2013]. Reporte s/n. Disponible en: http://www.senseaboutscience.org/data/files/IOP_MRI_Europe_version.pdf

Starn, R. (2008). The EMF Directive and protection of MRI Workers. Possible Solutions. BA Bilthoven (Utrecht): National Institute for Public Health and the Environment. Abril 2008 [citado 04 de junio de 2013]. Report 610703001/2008. Disponible en: http://www.rivm.nl/bibliotheek/rapporten/610703001.pdf

International Commission on Non-Ionizing Radiation Protection. (1998). Guidelines for limiting exposure to time-varying electric, magnetic and electromagnetic fields (up to $300 \mathrm{GHz}$ ). Health Physics 1998; 74: 494-522.

International Commission On Non-Ionizing Radiation Protection. (2003). Exposure to Static and Low Frequency Electromagnetic Fields, Biological Effects and Health Consequences (0-100 kHz), ICNIRP, Munich (2003)

Capstick M et al. (2008). Project VT/2007/017. An investigation into occupational exposure to electromagnetic fields for personnel working with and around medical magnetic resonance imaging equipment. www.myesr.org/html/img/ pool/VT2007017FinalReportv04.pdf.

Health Protection Agency. (2008). Static Magnetic fields.. Chilton: Health Protection Agency;2008. Report of the Independent Advisory Group on Non Ionising Radiation.

International Commission on Non- Ionizing Radiation Protection. (2004). Medical magnetic resonance (MR) procedures: protection of patients. Health Physics 2004; 87: 197-216. 
World Health Organization. (2006). Environmental Health Criteria 232-Static Fields. Pekin: OMS; 2006.

World Health Organization. (2007). Environmental Health Criteria 238-Extremely Low Frequency Fields. Madrid: OMS; 2007.

International Commission on Non-Ionizing Radiation Protection. (2009). Guidelines on Limits of Exposure to Static Magnetic Fields. Health Physics 2009; 96(4):504-514.

International Commission on Non-Ionizing Radiation Protection. (2009). Amendment to the ICNIRP Statement on Medical Magnetic Resonance (MR). Procedures: Protection of Patients" Health Physics 2009; 97(3):259-61.

International Commission on Non-Ionizing Radiation Protection. (2010). Guidelines for limiting exposure to time-varying electric and magnetic fields $(1 \mathrm{~Hz}$ - $100 \mathrm{kHz}$ ). Health Physics 2010; 99(6):818 -836.

International Electro technical Commission (2007). Particular requirements for the safety of magnetic resonance equipment for medical diagnosis. IEC std 60601-2-33 2nd edn 2nd amendment. (IEC: Geneva).

Institute of Electrical and Electronics Engineers. (2006). Standard for Safety Levels with Respect to Human Exposure Levels to Radio Frequency Electromagnetic Fields, $3 \mathrm{kHz}$ to $300 \mathrm{GHz}$, IEEE Standard C95.1. New York: IEEE; 2006.

Institute of Electrical and Electronics Engineers. (2006). Standard for Safety Levels with Respect to Human Exposure Levels to Radio Frequency Electromagnetic Fields, $3 \mathrm{kHz}$ to $300 \mathrm{GHz}$, IEEE Standard C95.1. New York: IEEE; 2006.

European Commission (2004). Directive 2004/40/EC of the European Parliament and of the Council of 29 April 2004 on the minimum health and safety requirements regarding the exposure of workers to the risks arising from physical agents (electromagnetic fields). Official Journal of the European Union L 159 of 30 April 2004, and corrigenda L 184 of 24 May 2004.

Directive 2008/46/EC of the European Parliament and of the Council (2008). Amending Directive 2004/40/EC on minimum health and safety requirements regarding the exposure of workers to the risks arising from physical agents (electromagnetic fields) Official Journal of the European Union L 114 of 26 April 2008.

Directiva 2012/11/UE del Parlamento Europeo y del Consejo (2012). Por la que se modifica la Directiva 2004/40/CE, sobre las disposiciones mínimas de seguridad y de salud relativas a la exposición de los trabajadores a los riesgos derivados de los agentes físicos (campos electromagnéticos) (decimoctava Directiva específica con arreglo al artículo 16 apartado 1, de la Directiva 89/391/CEE http://eur-lex. europa.eu/LexUriServ/LexUriServ.do?uri=OJ:L:2012:110:0001:0002:ES:PDF

Keevil, S. (2008). MRI and Physical Agents (EMF) Directive [Internet]. Londres: Institute of Physics; Nov 2008 [citado 3 de junio de 2013]. Reporte s/n. Disponible en: http://www.senseaboutscience.org/data/files/IOP MRI Europe version.pdf

Starn, R. (2008). The EMF Directive and protection of MRI Workers. Possible Solutions. BA Bilthoven (Utrecht): National Institute for Public Health and the 
Environment. Abril 2008 [citado 04 de junio de 2013]. Report 610703001/2008. Disponible en: http://www.rivm.nl/bibliotheek/rapporten/610703001.pdf

International Commission on Non-Ionizing Radiation Protection. (1998). Guidelines for limiting exposure to time-varying electric, magnetic and electromagnetic fields (up to $300 \mathrm{GHz}$ ). Health Physics 1998; 74: 494-522.

International Commission On Non-Ionizing Radiation Protection. (2003). Exposure to Static and Low Frequency Electromagnetic Fields, Biological Effects and Health Consequences (0-100 kHz), ICNIRP, Munich (2003)

Capstick M et al. (2008). Project VT/2007/017. An investigation into occupational exposure to electromagnetic fields for personnel working with and around medical magnetic resonance imaging equipment. www.myesr.org/html/img/ pool/VT2007017FinalReportv04.pdf

Health Protection Agency. (2008). Static Magnetic fields.. Chilton: Health Protection Agency. Report of the Independent Advisory Group on Non Ionising Radiation.

International Commission on Non- Ionizing Radiation Protection. (2004). Medical magnetic resonance (MR) procedures: protection of patients. Health Physics 2004; 87: 197-216.

World Health Organization. (2006). Environmental Health Criteria 232-Static Fields. Pekin: OMS.

World Health Organization. (2007). Environmental Health Criteria 238-Extremely Low Frequency Fields. Madrid: OMS.

International Commission on Non-Ionizing Radiation Protection. (2009). Guidelines on Limits of Exposure to Static Magnetic Fields. Health Physics. 96(4):504-514.

International Commission on Non-Ionizing Radiation Protection. (2009). Amendment to the ICNIRP Statement on Medical Magnetic Resonance (MR). Procedures: Protection of Patients" Health Physics. 97(3):259-61.

International Commission on Non-Ionizing Radiation Protection. (2010). Guidelines for limiting exposure to time-varying electric and magnetic fields $(1 \mathrm{~Hz}$ - $100 \mathrm{kHz}$ ). Health Physics. 99(6):818 -836.

International Electro technical Commission. (2007). Particular requirements for the safety of magnetic resonance equipment for medical diagnosis. IEC std 60601-2-33 2nd edn 2nd amendment. (IEC: Geneva).

Institute of Electrical and Electronics Engineers. (2006). Standard for Safety Levels with Respect to Human Exposure Levels to Radio Frequency Electromagnetic Fields, $3 \mathrm{kHz}$ to $300 \mathrm{GHz}$, IEEE Standard C95.1. New York: IEEE.

Institute of Electrical and Electronics Engineers. (2006). Standard for Safety Levels with Respect to Human Exposure Levels to Radio Frequency Electromagnetic Fields, $3 \mathrm{kHz}$ to $300 \mathrm{GHz}$, IEEE Standard C95.1. New York: IEEE. 